\title{
Polynomial approximations and universality
}

\author{
by
}

A. Mouze (Lille)

\begin{abstract}
We give another version of the recently developed abstract theory of universal series to exhibit a necessary and sufficient condition of polynomial approximation type for the existence of universal elements. This certainly covers the case of simultaneous approximation with a sequence of continuous linear mappings. In the case of a sequence of unbounded operators the same condition ensures existence and density of universal elements. Several known results, stronger statements or new results can be deduced in a unified way.
\end{abstract}

1. Introduction. The theory of universal series dates back almost a century and during that period several results concerning various types of these series have been obtained (see for instance [11, [8, [9] and the references therein). We refer to [7] for an excellent survey on universality. Recently Bayart, Grosse-Erdmann, Nestoridis and Papadimitropoulos have developed an abstract framework for the theory of universal series, from which they deduce in a unified way most of the known results [10, [2]. Let $A$ be a complete metrizable vector subspace of $\mathbb{K}^{\mathbb{N}}$. It turns out that the existence of universal elements in $A$ is equivalent to the existence of a function which approximates both a given function in the space where the universal property holds and zero in the space $A$ where the universal function should live. The existence of such universal series also implies topological genericity of such elements. The present paper deals with a special case of this abstract theorem, where we impose a simultaneous approximation in $A$ using a sequence of continuous linear mapping $T_{n}: A \rightarrow A$. From this, we exhibit a necessary and sufficient condition of polynomial approximation type for the existence of universal elements $a \in A$ so that $T_{n} a$ is dense in $A$ and all approximations are realized with the same sequence of indices. Moreover, if $A$ is a separable infinite-dimensional Banach space, we prove that the same condition implies the existence and the density of universal series under a sequence of

2010 Mathematics Subject Classification: 30B30, 30E10, 30K05, 30K10, 41A65, $47 \mathrm{~B} 37$.

Key words and phrases: universal series, polynomial approximation, operators on special spaces. 
unbounded operators on $A$. As a special case, we can consider sequences $\left(T_{n}\right)$ of operators that are generated by a single linear operator $T$ via iteration, that is, $T_{n}=T^{n}$. Hence the existence of such elements implies the hypercyclicity of $T$ (a vector $a$ is said to be a hypercyclic vector for $T$ provided its orbit $\left\{T^{n} a: n \in \mathbb{N}\right\}$ is dense). Thus we deduce a hypercyclicity criterion for unbounded operators. Finally, all theses results allow us to deduce and easily unify several known results, stronger statements or new results.

\section{From abstract theory to polynomial approximation}

2.1. Introduction. The theory of universality can be embedded in a very general framework. Let $Y$ be a complete metrizable topological vector space and $Z$ a metrizable topological vector space, endowed with translation invariant metrics $d$ and $\rho$, respectively.

Definition 2.1. Let $M$ be a separable closed subspace of $Z$ and $L_{n}$ : $Y \rightarrow Z, n \in \mathbb{N}$, continuous linear mappings. An element $y \in Y$ belongs to the $\operatorname{class} \mathcal{U}(\mathcal{L} ; M)$ if $M \subset \overline{\left\{L_{n} y: n \in \mathbb{N}\right\}}$.

The most general theorem of the abstract theory is the following (Theorem 27 of [2]).

THEOREM $2.2([2])$. Under the above assumptions, if there exists a dense subset $Y_{0}$ of $Y$ such that, for every $y \in Y_{0},\left(L_{n} y\right)$ converges to an element in $M$, then the following are equivalent:

$(\alpha) \mathcal{U}(\mathcal{L} ; M) \neq \emptyset$.

( $\beta)$ For every $z \in M$ and $\varepsilon>0$, there exist $n \geq 0$ and $y \in Y$ such that

$$
\rho\left(L_{n} y, z\right)<\varepsilon \text { and } d(y, 0)<\varepsilon .
$$

$(\gamma) \mathcal{U}(\mathcal{L} ; M)$ is a dense $G_{\delta}$ subset of $Y$.

A more restricted form of the abstract theory with straightforward applications is as follows.

Let $X$ be a metrizable topological vector space over the field $\mathbb{K}=\mathbb{R}$ or $\mathbb{C}$. Let $\rho$ be a translation invariant metric compatible with the operations + and . of the vector space $X$. Let $\left(x_{k}\right)_{k=0}^{+\infty}$ be a fixed sequence in $X$. We are interested in restricted universal series. We fix a subspace $A$ of $\mathbb{K}^{\mathbb{N}}$ and assume that it carries a complete metrizable vector space topology induced by a translation invariant metric $d$, such that the following three properties hold:

$\left(P_{1}\right)$ the coordinate projections $A \rightarrow \mathbb{K}, a \mapsto a_{m}$, are continuous for all $m \in \mathbb{N}$,

$\left(P_{2}\right)$ the set of polynomials $G=\left\{a=\left(a_{n}\right)_{n \geq 0} \in \mathbb{K}^{\mathbb{N}}:\left\{n: a_{n} \neq 0\right\}\right.$ is finite is contained in $A$,

$\left(P_{3}\right) G$ is dense in $A$. 
As usual we denote by $\left(e_{n}\right)_{n \geq 0}$ the canonical basis of $\mathbb{K}^{\mathbb{N}}$.

Definition 2.3. A sequence $a \in A$ belongs to the class $\mathcal{U}_{A}$ if, for every $x \in X$, there exists a sequence $\left(\lambda_{n}\right)$ in $\mathbb{N}$ such that

$$
\sum_{j=0}^{\lambda_{n}} a_{j} x_{j} \rightarrow x \quad \text { and } \quad \sum_{j=0}^{\lambda_{n}} a_{j} e_{j} \rightarrow a \quad \text { as } n \rightarrow+\infty .
$$

Observe that $\mathcal{U}_{A}=\mathcal{U}(\mathcal{L} ; X \times\{0\})$ with the map $L_{n}: A \rightarrow X \times A, a \mapsto$ $\left(\sum_{j=0}^{n} a_{j} x_{j}, \sum_{j=0}^{n} a_{j} e_{j}-a\right)$. We set $Y_{0}=G$, which is a dense subset of $A=Y$.

In this case, a condition of polynomial approximation type is equivalent to the existence of universal elements. Indeed, Theorem 2.2 takes the following form.

COROLlaRY 2.4 ([2]). Under the above assumptions the following are equivalent:

(1) $\mathcal{U}_{A} \neq \emptyset$.

(2) For every $x \in X$ and $\varepsilon>0$, there exist $n \in \mathbb{N}$ and $a_{0}, a_{1}, \ldots, a_{n} \in \mathbb{K}$ such that

$$
\rho\left(\sum_{j=0}^{n} a_{j} x_{j}, x\right)<\varepsilon \quad \text { and } \quad d\left(\sum_{j=0}^{n} a_{j} e_{j}, 0\right)<\varepsilon .
$$

(3) $\mathcal{U}_{A}$ is a dense $G_{\delta}$ subset of $A$.

2.2. Polynomial approximation and continuous mappings I. Let us now consider a sequence $T=\left(T_{n}\right)_{n \in \mathbb{N}}$ of continuous linear mappings $T_{n}: A \rightarrow A$, such that for every $a \in G,\left(T_{n} a\right)$ converges to an element in $A$.

Definition 2.5. An element $a \in A$ belongs to the class $\mathcal{U}_{A, T}$ if, for every $x \in X$ and $b \in A$, there exists an increasing sequence $\left(\lambda_{n}\right)$ of positive integers such that

$$
\rho\left(\sum_{j=0}^{\lambda_{n}} a_{j} x_{j}, x\right) \rightarrow 0, \quad d\left(\sum_{j=0}^{\lambda_{n}} a_{j} e_{j}, a\right) \rightarrow 0, \quad d\left(T_{\lambda_{n}}(a), b\right) \rightarrow 0
$$

as $n \rightarrow+\infty$. In this situation, Theorem 2.2 becomes

COROLlary 2.6. Under the above assumptions the following are equivalent:

(i) $\mathcal{U}_{A, T} \neq \emptyset$.

(ii) For every $x \in X, b \in A$ and $\varepsilon>0$, there exist $n, m \in \mathbb{N}$ with $m \geq n$ and $a_{0}, a_{1}, \ldots, a_{n}, a_{n+1}, \ldots, a_{m} \in \mathbb{K}$ such that

$$
\rho\left(\sum_{j=0}^{n} a_{j} x_{j}, x\right)<\varepsilon, \quad d\left(\sum_{j=0}^{n} a_{j} e_{j}, 0\right)<\varepsilon,
$$




$$
d\left(\sum_{j=n+1}^{m} a_{j} e_{j}, 0\right)<\varepsilon, \quad d\left(T_{n}\left(\sum_{j=0}^{m} a_{j} e_{j}\right), b\right)<\varepsilon .
$$

(iii) $\mathcal{U}_{A, T}$ is a dense $G_{\delta}$ subset of $A$.

Proof. Observe that $\mathcal{U}_{A, T}=\mathcal{U}(\mathcal{L} ; X \times A \times\{0\})$ with $L_{n}: \mathbb{K}^{\mathbb{N}} \rightarrow X \times$ $A \times A, a \mapsto\left(\sum_{j=0}^{n} a_{j} x^{j}, T_{n}(a), \sum_{j=0}^{n} a_{j} e_{j}-a\right)$. Then it suffices to prove that condition $(\beta)$ of Theorem 2.2 is equivalent to condition (ii).

$(\beta) \Rightarrow(\mathrm{ii})$ : Assume that, for every $z=(x, b, 0) \in X \times A \times\{0\}$ and $\varepsilon>0$, there exist $n \geq 0$ and $y \in A$ such that

$$
\begin{aligned}
& \rho\left(\sum_{j=0}^{n} y_{j} x_{j}, x\right)<\varepsilon / 2, \quad d\left(T_{n}(y), b\right)<\varepsilon / 2, \\
& d\left(\sum_{j=0}^{n} y_{j} e_{j}, y\right)<\varepsilon / 2, \quad d(y, 0)<\varepsilon / 4 .
\end{aligned}
$$

Using the fact that $G$ is dense in $A$ and $L_{n}$ is a continuous map, it follows immediately that there exists a polynomial $a=\sum_{j=0}^{m} a_{j} e_{j}$, with $m \geq n$, such that $d(a, y)<\varepsilon / 4$ and

$$
\begin{gathered}
\rho\left(\sum_{j=0}^{n} y_{j} x_{j}, \sum_{j=0}^{n} a_{j} x_{j}\right)<\varepsilon / 2, \quad d\left(T_{n}(y), T_{n}(a)\right)<\varepsilon / 2, \\
d\left(\sum_{j=0}^{n} y_{j} e_{j}, \sum_{j=0}^{n} a_{j} e_{j}\right)<\varepsilon / 4 .
\end{gathered}
$$

By using the triangle inequality, we get

$$
\begin{aligned}
\rho\left(\sum_{j=0}^{n} a_{j} x_{j}, x\right) & <\varepsilon / 2+\varepsilon / 2=\varepsilon, \quad d\left(T_{n}(a), b\right)<\varepsilon / 2+\varepsilon / 2=\varepsilon, \\
d\left(\sum_{j=n+1}^{m} a_{j} e_{j}, 0\right) & \leq d(a, y)+d\left(y, \sum_{j=0}^{n} y_{j} e_{j}\right)+d\left(\sum_{j=0}^{n} y_{j} e_{j}, \sum_{j=0}^{n} a_{j} e_{j}\right) \\
& <\varepsilon / 4+\varepsilon / 2+\varepsilon / 4=\varepsilon, \\
d\left(\sum_{j=0}^{n} a_{j} e_{j}, 0\right) & \leq d\left(\sum_{j=0}^{n} y_{j} e_{j}, \sum_{j=0}^{n} a_{j} e_{j}\right)+d\left(\sum_{j=0}^{n} y_{j} e_{j}, y\right)+d(y, 0) \\
& <\varepsilon / 4+\varepsilon / 2+\varepsilon / 4=\varepsilon .
\end{aligned}
$$

(ii) $\Rightarrow(\beta)$ : This is trivial.

2.3. Polynomial approximation and continuous mappings II. As in the abstract theory developed in [2], we need a small step forward in the abstract theory to cover the case of universal Taylor series. Let us first introduce the following formalism. We keep the notations $X$ and $G$. 
Denote by $E$ a complete metrizable topological vector space, with topology induced by a translation invariant metric $d$. Let $\left(\tilde{e}_{k}\right)_{k=0}^{+\infty}$ be a fixed sequence in $E$ and suppose that, for any $n \in \mathbb{N}$, we have a continuous linear map $\phi_{n}: E \rightarrow \mathbb{K}$. For every $a \in G$, we consider the polynomial $g_{a}=\sum_{j=0}^{+\infty} a_{j} \tilde{e}_{j}$ (which belongs to $E$ ) and we set $\operatorname{deg}\left(g_{a}\right)=\max \left\{j \in \mathbb{N}: a_{j} \neq 0\right\}$. We shall make the following assumptions.

$\left(E_{1}\right)$ The set $\left\{g_{a}: a \in G\right\}$ is dense in $E$.

$\left(E_{2}\right)$ For every $a \in G$ and every $j \in \mathbb{N}, \phi_{j}\left(g_{a}\right)=a_{j}$.

Let $T=\left(T_{n}\right)_{n \in \mathbb{N}}$ be a sequence of continuous linear mappings $T_{n}: E \rightarrow E$ such that, for every $a \in G,\left(T_{n}\left(g_{a}\right)\right)$ converges to an element in $E$.

Definition 2.7. An element $f \in E$ belongs to the class $\mathcal{U}_{E, T}$ if, for every $x \in X$ and $y \in E$, there exists an increasing sequence $\left(\lambda_{n}\right)$ of positive integers such that $\rho\left(\sum_{j=0}^{\lambda_{n}} \phi_{j}(f) x_{j}, x\right) \rightarrow 0, d\left(\sum_{j=0}^{\lambda_{n}} \phi_{j}(f) \tilde{e}_{j}, f\right) \rightarrow 0$ and $d\left(T_{\lambda_{n}}(f), y\right) \rightarrow 0$ as $n \rightarrow+\infty$.

Hence we have the following extension of Corollary 2.6, whose proof is similar.

COROLlaRY 2.8. Under the above assumptions the following are equivalent:

(i) $\mathcal{U}_{E, T} \neq \emptyset$.

(ii) For every $x \in X, y \in E$ and $\varepsilon>0$, there exist $n, m \in \mathbb{N}$ with $m \geq n$ and $a_{0}, a_{1}, \ldots, a_{n}, a_{n+1}, \ldots, a_{m} \in \mathbb{K}$ such that

$$
\begin{gathered}
\rho\left(\sum_{j=0}^{n} a_{j} x_{j}, x\right)<\varepsilon, \quad d\left(\sum_{j=0}^{n} a_{j} \tilde{e}_{j}, 0\right)<\varepsilon, \\
d\left(\sum_{j=n+1}^{m} a_{j} \tilde{e}_{j}, 0\right)<\varepsilon, \quad d\left(T_{n}\left(\sum_{j=0}^{m} a_{j} \tilde{e}_{j}\right), y\right)<\varepsilon .
\end{gathered}
$$

(iii) $\mathcal{U}_{E, T}$ is a dense $G_{\delta}$ subset of $E$.

As in Section 1.3 of [2], it is possible to have universality not only in a single set $X$, but also in a countable union of such sets. Hence we assume that $\left(X_{k}\right)_{k \geq 1}$ is a sequence of metrizable topological vector spaces over $\mathbb{K}$, equipped with translation invariant metrics $\rho_{k}$. For any $k \geq 1$, let $\left(x_{k, j}\right)_{j=0}^{+\infty}$ be a fixed sequence in $X_{k}$. We keep the same assumptions $\left(E_{i}\right), i=1,2$, with trivial modifications.

Definition 2.9. An element $f \in E$ belongs to the class $\mathcal{U}_{E, T}$ if, for every $k \geq 1$, every $x \in X_{k}$ and $y \in E$, there exists an increasing sequence $\left(\lambda_{n}\right)$ of positive integers such that $\rho_{k}\left(\sum_{j=0}^{\lambda_{n}} \phi_{j}(f) x_{k, j}, x\right) \rightarrow 0, d\left(\sum_{j=0}^{\lambda_{n}} \phi_{j}(f) \tilde{e}_{j}, f\right)$ $\rightarrow 0$ and $d\left(T_{\lambda_{n}}(f), y\right) \rightarrow 0$ as $n \rightarrow+\infty$.

We obtain the following result. 
COROLlARY 2.10. Under the above assumptions the following are equivalent:

(i) $\mathcal{U}_{E, T} \neq \emptyset$.

(ii) For every $k \geq 1, x \in X_{k}, y \in E$ and $\varepsilon>0$, there exist $n, m \in \mathbb{N}$ with $m \geq n$ and $a_{0}, a_{1}, \ldots, a_{n}, a_{n+1}, \ldots, a_{m} \in \mathbb{K}$ such that

$$
\begin{aligned}
& \rho_{k}\left(\sum_{j=0}^{n} a_{j} x_{k, j}, x\right)<\varepsilon, \quad d\left(\sum_{j=0}^{n} a_{j} \tilde{e}_{j}, 0\right)<\varepsilon, \\
& d\left(\sum_{j=n+1}^{m} a_{j} \tilde{e}_{j}, 0\right)<\varepsilon, \quad d\left(T_{n}\left(\sum_{j=0}^{m} a_{j} \tilde{e}_{j}\right), y\right)<\varepsilon .
\end{aligned}
$$

(iii) $\mathcal{U}_{E, T}$ is a dense $G_{\delta}$ subset of $E$.

For the proof we notice that a combination of Corollary 2.8 and Baire's Theorem yields the implication (i) $\Rightarrow$ (ii). The rest is straightforward.

2.4. Polynomial approximation and unbounded operators. Observe first that condition (ii) of Corollary 2.6 can be replaced by another condition of polynomial approximation type.

LEMMA 2.11. Under the assumptions of Corollary 2.6, assertion (ii) is equivalent to the following condition:

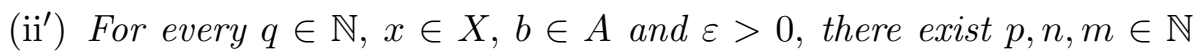
with $m \geq n \geq p \geq q$ and $a_{p}, a_{p+1}, \ldots, a_{n}, a_{n+1}, \ldots, a_{m} \in \mathbb{K}$ such that

$$
\begin{aligned}
& \rho\left(\sum_{j=p}^{n} a_{j} x_{j}, x\right)<\varepsilon, \quad d\left(\sum_{j=p}^{n} a_{j} e_{j}, 0\right)<\varepsilon, \quad d\left(\sum_{j=n+1}^{m} a_{j} e_{j}, 0\right)<\varepsilon, \\
& d\left(T_{n}\left(\sum_{j=p}^{m} a_{j} e_{j}\right), b\right)<\varepsilon, \quad d\left(T_{i}\left(\sum_{j=p}^{m} a_{j} e_{j}\right), 0\right)<\varepsilon, \quad i=1, \ldots, q .
\end{aligned}
$$

Proof. Indeed, $\left(\mathrm{ii}^{\prime}\right) \Rightarrow(\mathrm{ii})$ is trivial. The converse implication is not much more difficult: using the continuity of the mappings $T_{i}$, there exists $0<\varepsilon^{\prime}<$ $\varepsilon / 2$ such that for any $h \in A$ satisfying $d(h, 0)<\varepsilon^{\prime}$, we have $d\left(T_{i}(h), 0\right)<\varepsilon$, for every $i=1, \ldots, q$. Since condition (ii) implies the existence of universal elements, let $a \in \mathcal{U}_{A, T}$. Observe that, for any $a^{\prime} \in G$, we have $a+a^{\prime} \in \mathcal{U}_{A, T}$, thus there exists some $p \geq q$ so that $c=\left(0, \ldots, 0, a_{p}, a_{p+1}, \ldots\right)$ belongs to $\mathcal{U}_{A, T}$ and $d(c, 0)<\varepsilon^{\prime} / 2$. Since $c \in \mathcal{U}_{A, T}$, we may find $n>p$ such that

$$
\rho\left(\sum_{j=q}^{n} a_{j} x_{j}, x\right)<\varepsilon, \quad d\left(\sum_{j=p}^{n} a_{j} e_{j}, c\right)<\varepsilon / 2, \quad d\left(T_{n}(c), b\right)<\varepsilon / 2 .
$$

Since the operator $T_{n}$ is continuous, there exists $0<\varepsilon^{\prime \prime}<\varepsilon^{\prime} / 2$ such that for any $h \in A$ the inequality $d(c, h)<\varepsilon^{\prime \prime}$ implies $d\left(T_{n}(c), T_{n}(h)\right)<\varepsilon^{\prime}$. Since 
$c \in \mathcal{U}_{A, T}$, we may find $m>n$ such that $d\left(\sum_{j=p}^{m} a_{j} e_{j}, c\right)<\varepsilon^{\prime \prime}$. It follows $d\left(T_{n}\left(\sum_{j=p}^{m} a_{j} e_{j}\right), T_{n}(c)\right)<\varepsilon^{\prime}$. By the triangle inequality, we obtain

$$
\begin{aligned}
d\left(T_{n}\left(\sum_{j=p}^{m} a_{j} e_{j}\right), b\right) & \leq d\left(T_{n}\left(\sum_{j=p}^{m} a_{j} e_{j}\right), T_{n}(c)\right)+d\left(T_{n}(c), b\right)<\varepsilon^{\prime}+\varepsilon / 2<\varepsilon, \\
d\left(\sum_{j=p}^{n} a_{j} e_{j}, 0\right) & \leq d\left(\sum_{j=p}^{n} a_{j} e_{j}, c\right)+d(c, 0)<\varepsilon / 2+\varepsilon^{\prime} / 2<\varepsilon \\
d\left(\sum_{j=n+1}^{m} a_{j} e_{j}, 0\right) & \leq d\left(\sum_{j=p}^{m} a_{j} e_{j}, c\right)+d\left(\sum_{j=p}^{n} a_{j} e_{j}, c\right)<\varepsilon^{\prime \prime}+\varepsilon / 2<\varepsilon .
\end{aligned}
$$

Moreover,

$$
d\left(\sum_{j=p}^{m} a_{j} e_{j}, 0\right) \leq d\left(\sum_{j=p}^{m} a_{j} e_{j}, c\right)+d(c, 0)<\varepsilon^{\prime \prime}+\varepsilon^{\prime} / 2<\varepsilon^{\prime},
$$

which implies $d\left(T_{i}(h), 0\right)<\varepsilon$ for every $i=1,2, \ldots, q$. Therefore we have (ii').

Clearly we can replace conditions (ii) of Corollaries 2.8 and 2.10 by (ii') with obvious modifications.

Assume now that $A$ is a separable infinite-dimensional Banach space and let $\left(T_{n}\right)$ be a sequence of densely defined and closed linear operators. We keep the notations $X, G$ of the previous sections and we suppose that $G$ is a dense subset of the domain of $T_{n}$ for all positive integers $n$, such that for every $a \in G,\left(T_{n} a\right)$ converges to an element in $A$. Then condition (ii') implies the density of the set $\mathcal{U}_{A, T}$ (see Definition 2.5) of universal elements.

ThEOREM 2.12. Let $\left(T_{n}\right)$ be a sequence of densely defined and closed linear operators and suppose that $G$ is a dense subset of the domain of $T_{n}$ for all positive integers $n$, and that for every $a \in G,\left(T_{n} a\right)$ converges to an element in A. Assume that

(ii') for every $q \in \mathbb{N}, x \in X, b \in A$ and $\varepsilon>0$, there exist $p, n, m \in \mathbb{N}$ with $m \geq n \geq p \geq q$ and $a_{p}, a_{p+1} \ldots, a_{n}, a_{n+1}, \ldots, a_{m} \in \mathbb{K}$ such that

$$
\begin{gathered}
\rho\left(\sum_{j=p}^{n} a_{j} x_{j}, x\right)<\varepsilon, \quad\left\|\sum_{j=p}^{n} a_{j} e_{j}\right\|<\varepsilon, \quad\left\|\sum_{j=n+1}^{m} a_{j} e_{j}\right\|<\varepsilon, \\
\left\|T_{n}\left(\sum_{j=p}^{m} a_{j} e_{j}\right)-b\right\|<\varepsilon, \quad\left\|T_{i}\left(\sum_{j=p}^{m} a_{j} e_{j}\right)\right\|<\varepsilon, \quad i=1, \ldots, q .
\end{gathered}
$$

Then $\mathcal{U}_{A, T}$ is a dense subset of $A$. 
Proof. If we take $a_{j}$ in $\mathbb{Q}$ or in $\mathbb{Q}+i \mathbb{Q}$, the assumption implies that $X$ is separable. Let $\left(y_{s}\right)$ (resp. $\left(\tilde{y}_{t}\right)$ ) be a dense sequence in $X$ (resp. in $A$ ). Let us consider an enumeration $\left(y_{s_{i}}, \tilde{y}_{t_{i}}\right)$ of all pairs $\left(y_{s}, \tilde{y}_{t}\right)$. For notational convenience, we will just write $\left(y_{l}, \tilde{y}_{l}\right)$ instead of $\left(y_{s_{i}}, \tilde{y}_{t_{i}}\right)$. Let $h=\sum_{j \geq 0} h_{j} e_{j}$ be in $G$ and $\varepsilon>0$.

Step 1: There exists $u_{1} \in A$ such that $T_{n}(h) \rightarrow u_{1}$ as $n \rightarrow+\infty$. There exists an integer $q_{1}>\max \left\{j: h_{j} \neq 0\right\}$ such that $\left\|T_{n}(h)-u_{1}\right\|<1 / 2^{2}$ for every $n \geq q_{1}$. By assumption, there exist $p_{1}, n_{1}, m_{1} \in \mathbb{N}$ with $m_{1} \geq n_{1} \geq$ $p_{1} \geq q_{1}$ and $a_{p_{1}}, a_{p_{1}+1}, \ldots, a_{n_{1}}, a_{n_{1}+1}, \ldots, a_{m_{1}} \in \mathbb{K}$ such that

$$
\begin{gathered}
\rho\left(\sum_{j=p_{1}}^{n_{1}} a_{j} x_{j}, y_{1}-h\right)<1 / 2^{2}, \quad\left\|\sum_{j=p_{1}}^{n_{1}} a_{j} e_{j}\right\|<\varepsilon / 2^{2}, \\
\left\|\sum_{j=n_{1}+1}^{m_{1}} a_{j} e_{j}\right\|<\varepsilon / 2^{2}, \quad\left\|T_{n_{1}}\left(\sum_{j=p_{1}}^{m_{1}} a_{j} e_{j}\right)-\tilde{y}_{1}+u_{1}\right\|<1 / 2^{2} .
\end{gathered}
$$

Step 2: There exists $u_{2} \in A$ such that $T_{n}\left(h+\sum_{j=p_{1}}^{m_{1}} a_{j} e_{j}\right) \rightarrow u_{2}$ as $n \rightarrow+\infty$. We may find $q_{2} \geq m_{1}+1$ such that $\left\|T_{n}\left(h+\sum_{j=p_{1}}^{m_{1}} a_{j} e_{j}\right)-u_{2}\right\|<$ $1 / 2^{3}$ for every $n \geq q_{2}$. By assumption, there exist $p_{2}, n_{2}, m_{2} \in \mathbb{N}$ with $m_{2} \geq n_{2} \geq p_{2} \geq q_{2}$ and $a_{p_{2}}, a_{p_{2}+1}, \ldots, a_{n_{2}}, a_{n_{2}+1}, \ldots, a_{m_{2}} \in \mathbb{K}$ such that

$$
\begin{gathered}
\rho\left(\sum_{j=p_{2}}^{n_{2}} a_{j} x_{j}, y_{2}-h-\sum_{j=p_{1}}^{m_{1}} a_{j} x_{j}\right)<1 / 2^{3}, \quad\left\|\sum_{j=p_{2}}^{n_{2}} a_{j} e_{j}\right\|<\varepsilon / 2^{3}, \\
\left\|\sum_{j=n_{2}+1}^{m_{2}} a_{j} e_{j}\right\|<\varepsilon / 2^{3}, \quad\left\|T_{n_{2}}\left(\sum_{j=p_{2}}^{m_{2}} a_{j} e_{j}\right)-\tilde{y}_{2}+u_{2}\right\|<1 / 2^{3}, \\
\left\|T_{n_{1}}\left(\sum_{j=p_{2}}^{m_{2}} a_{j} e_{j}\right)\right\|<1 / 2^{3} .
\end{gathered}
$$

Assume that, for every $i=1, \ldots, l-1$, the integers $m_{i} \geq n_{i} \geq p_{i} \geq q_{i}$ and the coefficients $a_{p_{i}}, \ldots, a_{m_{i}}$ are constructed.

Step l: There exists $u_{l} \in A$ such that $T_{n}\left(h+\sum_{i=1}^{l-1} \sum_{j=p_{i}}^{m_{i}} a_{j} e_{j}\right) \rightarrow u_{l}$ as $n \rightarrow+\infty$. We find $q_{l} \geq m_{l-1}+1$ such that $\left\|T_{n}\left(h+\sum_{i=1}^{l-1} \sum_{j=p_{i}}^{m_{i}} a_{j} e_{j}\right)-u_{l}\right\|$ $<1 / 2^{l+1}$ for every $n \geq q_{l}$. By assumption, there exist $p_{l}, n_{l}, m_{l} \in \mathbb{N}$ with $m_{l} \geq n_{l} \geq p_{l} \geq q_{l}$ and $a_{p_{l}}, a_{p_{l}+1}, \ldots, a_{n_{l}}, a_{n_{l}+1}, \ldots, a_{m_{l}} \in \mathbb{K}$ such that

$$
\begin{gathered}
\rho\left(\sum_{j=p_{l}}^{n_{l}} a_{j} x_{j}, y_{l}-h-\sum_{i=1}^{l-1} \sum_{j=p_{i}}^{m_{i}} a_{j} x_{j}\right)<1 / 2^{l+1}, \quad\left\|\sum_{j=p_{l}}^{n_{l}} a_{j} e_{j}\right\|<\varepsilon / 2^{l+1}, \\
\left\|\sum_{j=n_{l}+1}^{m_{l}} a_{j} e_{j}\right\|<\varepsilon / 2^{l+1}, \quad\left\|T_{n_{l}}\left(\sum_{j=p_{l}}^{m_{l}} a_{j} e_{j}\right)-\tilde{y}_{l}+u_{l}\right\|<1 / 2^{l+1},
\end{gathered}
$$




$$
\left\|T_{n_{i}}\left(\sum_{j=p_{l}}^{m_{l}} a_{j} e_{j}\right)\right\|<1 / 2^{l+1}, \quad i=1, \ldots, l-1 .
$$

We set $a=h+\sum_{i=1}^{+\infty} \sum_{j=p_{i}}^{m_{i}} a_{j} e_{j}=\sum_{j=0}^{+\infty} a_{j} e_{j}$. Clearly we get

$$
\|a-h\| \leq \sum_{i=1}^{+\infty}\left\|\sum_{j=p_{i}}^{m_{i}} a_{j} e_{j}\right\|<\sum_{i=1}^{+\infty} \varepsilon / 2^{i}=\varepsilon, \quad \text { so } \quad\|a\| \leq\|h\|+\varepsilon
$$

Let $x \in X, b \in A$ and $\eta>0$. We will find $N \in \mathbb{N}$ so that $\rho\left(\sum_{j=0}^{N} a_{j} e_{j}, x\right)<\eta$, $\left\|\sum_{j=0}^{N} a_{j} e_{j}-a\right\|<\eta$ and $\left\|T_{N}(a)-b\right\|<\eta$. First we let $l \geq 1$ be a fixed integer and observe that

$$
\begin{aligned}
\left(\sum_{i=l+1}^{M} \sum_{j=p_{i}}^{m_{i}} a_{j} e_{j}, T_{n_{l}}\left(\sum_{i=l+1}^{M} \sum_{j=p_{i}}^{m_{i}} a_{j} e_{j}\right)\right) & =\left(\sum_{i=l+1}^{M} \sum_{j=p_{i}}^{m_{i}} a_{j} e_{j}, \sum_{i=l+1}^{M} T_{n_{l}}\left(\sum_{j=p_{i}}^{m_{i}} a_{j} e_{j}\right)\right) \\
& \rightarrow\left(\sum_{i=l+1}^{+\infty} \sum_{j=p_{i}}^{m_{i}} a_{j} e_{j}, \sum_{i=l+1}^{+\infty} T_{n_{l}}\left(\sum_{j=p_{i}}^{m_{i}} a_{j} e_{j}\right)\right)
\end{aligned}
$$

as $M \rightarrow+\infty$. Since $T_{n_{l}}$ is closed, the vector $\sum_{i=l+1}^{+\infty} \sum_{j=p_{i}}^{m_{i}} a_{j} e_{j}$ is in the domain of $T_{n_{l}}$ and

$$
T_{n_{l}}\left(\sum_{i=l+1}^{+\infty} \sum_{j=p_{i}}^{m_{i}} a_{j} e_{j}\right)=\sum_{i=l+1}^{+\infty} T_{n_{l}}\left(\sum_{j=p_{i}}^{m_{i}} a_{j} e_{j}\right)
$$

It follows that $a$ belongs to the domain of $T_{n_{l}}$. Clearly we may find $l \in \mathbb{N}$ such that $\rho\left(x, y_{l}\right)<\eta / 2,\left\|b-\tilde{y}_{l}\right\|<\eta / 2$ and $2<2^{l} \eta$. By construction, we have $\rho\left(h+\sum_{i=1}^{l-1} \sum_{j=p_{i}}^{m_{i}} a_{j} e_{j}+\sum_{j=p_{l}}^{n_{l}} a_{j} e_{j}, y_{l}\right)<1 / 2^{l+1}$, which implies

$$
\rho\left(\sum_{j=0}^{n_{l}} a_{j} e_{j}, x\right) \leq \rho\left(\sum_{j=0}^{n_{l}} a_{j} e_{j}, y_{l}\right)+\rho\left(y_{l}, x\right)<1 / 2^{l+1}+\eta / 2 \leq \eta .
$$

Moreover,

$$
\begin{aligned}
\left\|a-\sum_{j=0}^{n_{l}} a_{j} e_{j}\right\| & =\left\|\sum_{j=n_{l+1}}^{m_{l}} a_{j} e_{j}+\sum_{i=l+1}^{+\infty} \sum_{j=p_{i}}^{m_{i}} a_{j} e_{j}\right\| \\
& \leq\left\|\sum_{j=n_{l+1}}^{m_{l}} a_{j} e_{j}\right\|+\sum_{i=l+1}^{+\infty}\left\|\sum_{j=p_{i}}^{m_{i}} a_{j} e_{j}\right\| \leq \varepsilon / 2^{l+1}+\varepsilon / 2^{l}<\eta .
\end{aligned}
$$

Finally, we get

(4) $\left\|T_{n_{l}}(a)-b\right\| \leq\left\|T_{n_{l}}\left(h+\sum_{i=1}^{l} \sum_{j=p_{i}}^{m_{i}} a_{j} e_{j}\right)-b\right\|+\left\|T_{n_{l}}\left(\sum_{i=l+1}^{+\infty} \sum_{j=p_{i}}^{m_{i}} a_{j} e_{j}\right)\right\|$. 
Combining (4) with the estimates

$$
\begin{aligned}
\left\|T_{n_{l}}\left(h+\sum_{i=1}^{l} \sum_{j=p_{i}}^{m_{i}} a_{j} e_{j}\right)-b\right\| \leq & \left\|T_{n_{l}}\left(h+\sum_{i=1}^{l-1} \sum_{j=p_{i}}^{m_{i}} a_{j} e_{j}\right)-u_{l}\right\| \\
& +\left\|T_{n_{l}}\left(\sum_{j=p_{l}}^{m_{l}} a_{j} e_{j}\right)-b+u_{l}\right\| \\
\leq & 1 / 2^{l+1}+1 / 2^{l+1}<\eta / 2
\end{aligned}
$$

and

$$
\left\|T_{n_{l}}\left(\sum_{i=l+1}^{+\infty} \sum_{j=p_{i}}^{m_{i}} a_{j} e_{j}\right)\right\| \leq \sum_{i=l+1}^{+\infty}\left\|T_{n_{l}}\left(\sum_{j=p_{i}}^{m_{i}} a_{j} e_{j}\right)\right\| \leq \sum_{i=l+1}^{+\infty} 1 / 2^{i}=1 / 2^{l}<\eta / 2,
$$

we deduce $\left\|T_{n_{l}}(a)-b\right\|<\eta$. This completes the proof.

As in Section 1.3 of [2], it is possible to have universality not only in a single set $X$, but also in a countable union of such sets. Hence we assume that $\left(X_{k}\right)_{k \geq 1}$ is a sequence of metrizable topological vector spaces over $\mathbb{K}$, equipped with translation invariant metrics $\rho_{k}$. For any $k \geq 1$, let $\left(x_{k, j}\right)_{j=0}^{+\infty}$ be a fixed sequence in $X_{k}$. Assume that there exists a dense sequence $\left(y_{s, k}\right)_{s=1}^{+\infty}$ in $X_{k}$, for every $k \geq 1$.

Definition 2.13. An element $a=\left(a_{0}, a_{1}, \ldots\right) \in A$ belongs to the class $\tilde{\mathcal{U}}_{A, T}$ if, for every $k \geq 1$, every $x \in X_{k}$ and $b \in A$, there exists an increasing sequence $\left(\lambda_{n}\right)$ of positive integers such that

$\rho_{k}\left(\sum_{j=0}^{\lambda_{n}} a_{j} x_{k, j}, x\right) \rightarrow 0, d\left(\sum_{j=0}^{\lambda_{n}} a_{j} \tilde{e}_{j}, a\right) \rightarrow 0, d\left(T_{\lambda_{n}}(a), b\right) \rightarrow 0 \quad$ as $n \rightarrow+\infty$.

THEOREM 2.14. Under the above assumptions, assume that

(ii') for every $q \in \mathbb{N}, k \geq 1, x \in X_{k}, b \in A$ and $\varepsilon>0$, there exist $p, n, m \in \mathbb{N}$ with $m \geq n \geq p \geq q$ and $a_{p}, a_{p+1}, \ldots, a_{n}, a_{n+1}, \ldots, a_{m}$ $\in \mathbb{K}$ such that

$$
\begin{gathered}
\rho_{k}\left(\sum_{j=p}^{n} a_{j} x_{k, j}, x\right)<\varepsilon, \quad\left\|\sum_{j=p}^{n} a_{j} e_{j}\right\|<\varepsilon, \quad\left\|\sum_{j=n+1}^{m} a_{j} e_{j}\right\|<\varepsilon, \\
\left\|T_{n}\left(\sum_{j=p}^{m} a_{j} e_{j}\right)-b\right\|<\varepsilon, \quad\left\|T_{i}\left(\sum_{j=p}^{m} a_{j} e_{j}\right)\right\|<\varepsilon, \quad i=1, \ldots, q .
\end{gathered}
$$

Then $\tilde{\mathcal{U}}_{A, T}$ is a dense subset of $A$.

Proof. Let $\left(\tilde{y}_{t}\right)$ be a dense sequence in $A$. Let $\left(y_{s_{i}, k_{i}}, \tilde{y}_{t_{i}}, \rho_{k_{i}}\right)$ be an enumeration of all triplets $\left(y_{s, k}, \tilde{y}_{t}, \rho_{k}\right)$. We proceed as in the proof of Theorem 2.12 . 
REMARK 2.15. Theorem 2.12 seems to give a new sufficient condition for the existence of hypercyclic vectors of an unbounded operator. Let us recall that a vector $a$ is hypercyclic for an unbounded operator $T$ on a separable infinite-dimensional Banach space $A$ if for every integer $n \geq 0, T^{n} a$ is in the domain of $T$ and the orbit $\left\{a, T a, T^{2} a, \ldots\right\}$ is dense in $A$. We deduce from Theorem 2.12 the following.

TheOREM 2.16. Let $A \subset \mathbb{K}^{\mathbb{N}}$ be a separable infinite-dimensional Banach space and let $T$ be a densely defined and closed linear operator on $A$ for which $T^{n}$ is a closed operator for all positive integers $n$. Suppose that the set of polynomials $G=\left\{a=\left(a_{n}\right)_{n \geq 0} \in \mathbb{K}^{\mathbb{N}}:\left\{n: a_{n} \neq 0\right\}\right.$ is finite $\}$ is a dense subset of the domain of $T^{n}$ for all positive integers $n$, and for every $a \in G,\left(T^{n} a\right)$ converges to an element in $A$. Then $T$ is hypercyclic if for every $q \in \mathbb{N}, b \in A$ and $\varepsilon>0$, there exist $p, n, m \in \mathbb{N}$ with $m \geq n \geq p \geq q$ and $a_{p}, a_{p+1} \ldots, a_{n}, a_{n+1}, \ldots, a_{m} \in \mathbb{K}$ such that

and

$$
\left\|\sum_{j=p}^{m} a_{j} e_{j}\right\|<\varepsilon, \quad\left\|T^{n}\left(\sum_{j=p}^{m} a_{j} e_{j}\right)-b\right\|<\varepsilon
$$

$$
\left\|T^{i}\left(\sum_{j=p}^{m} a_{j} e_{j}\right)\right\|<\varepsilon, \quad i=1, \ldots, q .
$$

We can compare this result with Theorem 2.1 of [3].

\section{Applications}

3.1. Universality and weighted backward shift. We set $E=A=\mathbb{C}^{\mathbb{N}}$ which is endowed with the classical metric $d(a, b)=\sum_{n=0}^{\infty}\left(1 / 2^{n}\right)\left(\left|a_{n}-b_{n}\right| /\right.$ $\left.\left(1+\left|a_{n}-b_{n}\right|\right)\right), a, b \in A$. We fix a compact set $K \subset \mathbb{C}$ such that $0 \notin K$ and $K^{c}$ is connected. Let $X=H(\mathbb{C})$ be the space of entire functions endowed with the metric $\rho(f, g)=\sum_{n=0}^{\infty}\left(1 / 2^{n}\right)\left(\|f-g\|_{n} /\left(1+\|f-g\|_{n}\right)\right)$, where $\|f-g\|_{n}=\sup _{K}\left|f^{(n)}-g^{(n)}\right|, f, g \in H(\mathbb{C})$. The metric spaces $A$ and $X$ satisfy our assumptions. We set $x_{j}=\left(z \mapsto z^{j}\right)$. Let

$$
T: A \rightarrow A, \quad a=\left(a_{0}, a_{1}, a_{2}, \ldots\right) \mapsto\left(a_{1}, 2 a_{2}, 3 a_{3}, \ldots\right) .
$$

For any $n \in \mathbb{N}^{*}$, we denote $T_{n}=T^{n}$.

Proposition 3.1. Let $K \subset \mathbb{C}$ be a compact set with $0 \notin K$ and $K^{c}$ connected. Let $T$ be the weighted backward shift defined by $T\left(a_{0}, a_{1}, a_{2}, a_{3}, \ldots\right)=$ $\left(a_{1}, 2 a_{2}, 3 a_{3}, \ldots\right)$ for $a \in \mathbb{C}^{\mathbb{N}}$. Then there exists a sequence $a=\left(a_{n}\right) \in \mathbb{C}^{\mathbb{N}}$ such that for every $b \in \mathbb{C}^{\mathbb{N}}$ and every entire function $h$ there exists $\left(\lambda_{n}\right) \in \mathbb{N}^{\mathbb{N}}$ such that for every $l \in \mathbb{N}$,

$$
T^{\lambda_{n}}(a) \rightarrow b \quad \text { and } \quad \sup _{z \in K}\left|\left(\sum_{j=0}^{\lambda_{n}} a_{j} z^{j}\right)^{(l)}-h^{(l)}(z)\right| \rightarrow 0 \quad \text { as } n \rightarrow+\infty .
$$


The set of such sequences is $G_{\delta}$ and dense in $\mathbb{C}^{\mathbb{N}}$ endowed with the Cartesian topology.

Proof. We prove that condition (ii) of Corollary 2.6 holds.

Let $p \in \mathbb{N}, \varepsilon>0, h \in X$ and $b \in A$. We fix $N \in \mathbb{N}$ such that $N>\max \left(p, \sup _{z \in K}|z|\right)$ and $\sum_{n=N+1}^{+\infty} 1 / 2^{n}<\varepsilon / 4$. Since $0 \notin K$ and $K^{c}$ is connected, we find a simply connected open set $\Omega$ such that $0 \notin \Omega$ and $K \subset \Omega$. By Runge's Theorem, there exists a sequence $\left(q_{k}\right)$ of polynomials converging to $h(z) / z^{N+1}$ uniformly on compact subsets of $\Omega$. We set $p_{k}(z)=z^{N+1} q_{k}(z)$. Clearly using Weierstrass' Theorem we obtain, for a large enough $k_{0}, \sup _{K}\left|p_{k_{0}}^{(l)}-h^{(l)}\right|<\varepsilon / 8$ for $l=0,1, \ldots, N$. We have $p_{k_{0}}=\sum_{j=N+1}^{n_{0}} a_{j} z^{j}$. Therefore we get

$d\left(\sum_{j=N+1}^{n_{0}} a_{j} e_{j}, 0\right)<\varepsilon / 4, \quad \rho\left(\sum_{j=N+1}^{n_{0}} a_{j} z^{j}, h\right) \leq \sum_{l=0}^{N} 2^{-l}\left\|p_{k_{0}}-h\right\|_{l}+\varepsilon / 4<\varepsilon / 2$.

Consider $b^{\prime}=\left(b_{0}, b_{1}, \ldots, b_{N}, 0, \ldots\right)$ and $k_{1}>n_{0}$ such that

$$
\left|b_{0}\right| N^{k_{1}-l} /\left(k_{1}-l\right) !<\varepsilon / 8 \quad \text { for } l=0,1, \ldots, N \text {. }
$$

We set

$$
b^{\prime \prime}=\left(0, \ldots, 0, \frac{b_{0}}{k_{1} !}, \frac{1 ! b_{1}}{\left(k_{1}+1\right) !}, \ldots, \frac{N ! b_{N}}{\left(k_{1}+N\right) !}, 0, \ldots\right),
$$

where $b_{k_{1}}^{\prime \prime}=b_{0} / k_{1}$ !. An easy calculation gives $T_{k_{1}}\left(b^{\prime \prime}\right)=b^{\prime}$ and $d\left(b^{\prime}, b\right)<\varepsilon / 4$. Denote

$$
\sum_{j=N+1}^{n_{0}} a_{j} e_{j}+\sum_{j=k_{1}}^{k_{1}+N} \frac{b_{j-k_{1}}}{j !} e_{j}=\sum_{j=p}^{m} a_{j} e_{j}
$$

upon setting $m=k_{1}+N, a_{j}=0$ for $p \leq j \leq N$ and for $n_{0}<j<k_{1}$. Let $n=k_{1}$; thus from (5) and (6), we get

$$
\rho\left(\sum_{j=p}^{n} a_{j} z^{j}, h\right) \leq \rho\left(\sum_{j=N+1}^{n_{0}} a_{j} z^{j}, h\right)+\rho\left(a_{n} z^{n}, 0\right)<\varepsilon / 2+\varepsilon / 2=\varepsilon .
$$

We also have $d\left(\sum_{j=p}^{n} a_{j} e_{j}, 0\right)<\varepsilon / 4$ and $d\left(\sum_{j=n+1}^{m} a_{j} e_{j}, 0\right)<\varepsilon / 4$. Moreover, taking into account the property $T_{n}\left(\sum_{j=p}^{n-1} a_{j} e_{j}\right)=0$, we obtain

$$
d\left(T_{n}\left(\sum_{j=p}^{m} a_{j} e_{j}\right), b\right)=d\left(T_{n}\left(\sum_{j=n}^{m} a_{j} e_{j}\right), b\right)=d\left(b^{\prime}, b\right)<\varepsilon / 4 .
$$

Hence condition (ii) of Corollary 2.6 is satisfied.

Let us recall that there exists a sequence $\left(K_{k}\right)$ of compact set of $\mathbb{C}$ with $0 \notin K_{k}$ and $K_{k}^{c}$ connected such that every compact set $K \subset \mathbb{C}$ with $0 \notin K$ 
and $K^{c}$ connected is contained in some $K_{k}$ (see [9]). We combine the previous result with Corollary 2.10 to obtain the following.

TheOREM 3.2. Let $T$ be the weighted backward shift defined by the equality $T\left(a_{0}, a_{1}, a_{2}, a_{3}, \ldots\right)=\left(a_{1}, 2 a_{2}, 3 a_{3}, \ldots\right)$ for $a \in \mathbb{C}^{\mathbb{N}}$. Then there exists a sequence $a=\left(a_{n}\right) \in \mathbb{C}^{\mathbb{N}}$ such that, for every $b \in \mathbb{C}^{\mathbb{N}}$, every entire function $h$ and every compact set $K \subset \mathbb{C}$ with $0 \notin K$ and $K^{c}$ connected, there exists $\left(\lambda_{n}\right) \in \mathbb{N}^{\mathbb{N}}$ such that, for every $l \in \mathbb{N}$,

$$
T^{\lambda_{n}}(a) \rightarrow b \quad \text { and } \quad \sup _{z \in K}\left|\left(\sum_{j=0}^{\lambda_{n}} a_{j} z^{j}\right)^{(l)}-h^{(l)}(z)\right| \rightarrow 0 \quad \text { as } n \rightarrow+\infty .
$$

The set of such sequences is $G_{\delta}$ and dense in $\mathbb{C}^{\mathbb{N}}$ endowed with the Cartesian topology.

REMARKS 3.3.

1. In the last theorem, we can require the approximation of partial sums for $l=0$ alone, and by Mergelyan's Theorem we can assume that $h \in A(K)$. This gives a special case of Theorem 2.8 of [6].

2. Proposition 3.1 and Theorem 3.2 are also valid if we replace $T$ by another weighted backward shift $B_{\omega}$ with $B_{\omega}\left(e_{j}\right)=\omega_{j-1} e_{j-1}$ for $j \geq 1$, whenever $\left(\omega_{j}\right)$ is a sequence of strictly positive real numbers satisfying $\left(\prod_{k=0}^{n-1} \omega_{k}\right)^{-1} C^{n} \rightarrow 0$ as $n \rightarrow+\infty$, for every $C>0$. Under these conditions, $B_{\omega}$ is obviously a continuous map and the same proof works.

3. In Theorem 3.2 and Proposition 3.1 the case $l=0$ implies the result for all $l=0,1,2, \ldots$ because $\mathbb{C} \backslash\{0\}$ is open.

3.2. Universal Taylor series under derivatives. As usual, we denote by $\hat{\mathbb{C}}=\mathbb{C} \cup\{\infty\}$ the Riemann sphere, and we now suppose that $\Omega$ is a simply connected domain in $\mathbb{C}$ such that $\hat{\mathbb{C}} \backslash \bar{\Omega}$ is connected. Now $A^{\infty}(\Omega)$ denotes the set of functions $f \in H(\Omega)$ such that each derivative $f^{(l)}$ extends continuously on $\bar{\Omega}$. We endow this space with the family of seminorms $\sup _{|z| \leq n, z \in \bar{\Omega}}\left|f^{(l)}(z)\right|, l, n \in \mathbb{N}$. Thus $A^{\infty}(\Omega)$ becomes a Fréchet space equipped with the standard invariant metric $d$. We denote by $E=X^{\infty}(\Omega)$ the closure of the set of polynomials in $A^{\infty}(\Omega)$. The space $\left(X^{\infty}(\Omega), d\right)$ is complete. Notice that there exists a sequence of compact sets $K_{k} \subset \mathbb{C}$ with $K_{k} \cap \bar{\Omega}=\emptyset, K_{k}^{c}$ connected, and such that every compact set $K \subset \mathbb{C}$ with $K \cap \bar{\Omega}=\emptyset$ and $K^{c}$ connected is contained in some $K_{k}$ [2]. We let $X_{k}, k \geq 1$, be the space of entire functions endowed with the standard metric $\rho_{k}$ induced by the seminorms $\sup _{z \in K_{k}}\left|f^{(l)}(z)\right|, l \geq 0$. Set $L_{n}=\bar{\Omega} \cap\{z:|z| \leq n\}$ and let $\zeta \in \Omega$ be a fixed point in $L_{1}$. Clearly $\bigcup_{n} L_{n}=\bar{\Omega}$. For $f \in E$ and $n \geq 0$, we set $\tilde{e}_{n}=\left(z \mapsto(z-\zeta)^{n}\right)=x_{k, n}$ and $\phi_{n}(f)=f^{(n)}(\zeta) / n$ !. Clearly 
the assumptions of Section 2.3 are satisfied. Let $T: E \rightarrow E, f \mapsto f^{\prime}$. For any $n \in \mathbb{N}^{*}$, we denote $T_{n}=T^{n}$.

THEOREM 3.4. There exists a holomorphic function $f \in A^{\infty}(\Omega)$ such that, if we denote $S_{N}(f, \zeta)(z)=\sum_{n=0}^{N}\left(f^{(n)}(\zeta) / n !\right)(z-\zeta)^{n}$, the following property holds: for every function $g \in X^{\infty}(\Omega)$, every entire function $h$ and every compact set $K \subset \mathbb{C}$ with $K \cap \bar{\Omega}=\emptyset$ and $K^{c}$ connected, there exists an increasing sequence $\left(\lambda_{n}\right) \in \mathbb{N}^{\mathbb{N}}$ such that for every compact set $L \subset \Omega$ and every $l \in \mathbb{N}$, we have

$$
\begin{aligned}
\sup _{z \in L}\left|f^{\left(\lambda_{n}+l\right)}(z)-g^{(l)}(z)\right| \rightarrow 0 & \text { as } n \rightarrow+\infty, \\
\sup _{z \in K}\left|S_{\lambda_{n}}^{(l)}(f, \zeta)(z)-h^{(l)}(z)\right| \rightarrow 0 & \text { as } n \rightarrow+\infty .
\end{aligned}
$$

The set of such $f$ is $G_{\delta}$ and dense in $X^{\infty}(\Omega)$.

Proof. It suffices to prove that condition (ii) of Corollary 2.10 holds. Let $k \geq 0, p \in \mathbb{N}, \varepsilon>0, x \in X_{k}$ and $y \in E$. We fix $N \in \mathbb{N}$ such that $N>p$ and $\sum_{n=N+1}^{+\infty} 1 / 2^{n}<\varepsilon / 4$. As in the proof of Theorem 6 of [2], we construct a polynomial $P(z)=\sum_{j=N+1}^{n_{0}} a_{j}(z-\zeta)^{j}$ satisfying

$$
\begin{aligned}
& \sup _{z \in K_{k}}\left|P^{(l)}(z)-x^{(l)}(z)\right|<\varepsilon / 8, \quad \\
& \sup _{z \in L_{N}}\left|P^{(l)}(z)\right|<\varepsilon / 8,
\end{aligned}
$$

Since $y \in X^{\infty}(\Omega)$, the closure of the set of polynomials in $A^{\infty}(\Omega)$, there exists a polynomial $q(z)=\sum_{j=0}^{n_{1}} q_{j}(z-\zeta)^{j}$ such that

$$
\sup _{L_{N}}\left|q^{(l)}-y^{(l)}\right|<\varepsilon / 4, \quad l=0,1, \ldots, N .
$$

Take $R>\max \left(1, \sup _{z \in K_{k} \cup L_{N}}|z-\zeta|\right)$. We set

$$
q_{k_{1}}(z)=\sum_{j=0}^{n_{1}}\left(j ! q_{j} /\left(j+k_{1}\right) !\right)(z-\zeta)^{j+k_{1}}
$$

and choose $k_{1}>n_{0}$ such that for $l=0,1, \ldots, N$,

$$
\sum_{i=0}^{n_{1}}\left(i !\left|q_{i}\right| /\left(i+k_{1}-l\right) !\right) R^{i+k_{1}-l}<\varepsilon / 8 .
$$

Denote $\sum_{j=N+1}^{n_{0}} a_{j}(z-\zeta)^{j}+\sum_{j=k_{1}}^{n_{1}+k_{1}}\left(q_{j-k_{1}} / j !\right)(z-\zeta)^{j}=\sum_{j=p}^{m} a_{j}(z-\zeta)^{j}$, upon setting $m=k_{1}+n_{1}, a_{j}=0$ for $p \leq j \leq N$ and for $n_{0}<j<k_{1}$. We set $n=k_{1}$ and we end the proof as in Proposition 3.1. Indeed, we have 


$$
\begin{aligned}
& \rho_{k}\left(\sum_{j=p}^{n} a_{j}(z-\zeta)^{j}, x\right) \leq \rho_{k}\left(\sum_{j=p}^{n_{0}} a_{j}(z-\zeta)^{j}, x\right)+\rho_{k}\left(q_{0}(z-\zeta)^{k_{1}} / k_{1} !, 0\right) \\
&<\varepsilon / 2+\varepsilon / 2=\varepsilon \\
& d\left(\sum_{j=p}^{n} a_{j}(z-\zeta)^{j}, 0\right) \leq d\left(\sum_{j=p}^{n_{0}} a_{j}(z-\zeta)^{j}, 0\right)+d\left(q_{0}(z-\zeta)^{k_{1}} / k_{1} !, 0\right) \\
&<\varepsilon / 2+\varepsilon / 2=\varepsilon \\
& d\left(\sum_{j=n+1}^{m} a_{j}(z-\zeta)^{j}, 0\right)=d\left(\sum_{j=1}^{n_{1}} a_{j}(z-\zeta)^{j}, 0\right)<\varepsilon / 2 \\
& d\left(T_{n}\left(\sum_{j=p}^{m} a_{j}(z-\zeta)^{j}\right), y\right)=d(q, y)<\varepsilon . \mathbf{m}
\end{aligned}
$$

REMARKS 3.5.

1. We refer the reader to [4] or [2] for results of the same type as Theorem 3.4 .

2. In Theorem 3.4, we can require the approximation for $l=0$ alone and we can choose a compact set $L \subset \Omega$ with $L^{c}$ connected. Thus, by Mergelyan's Theorem, we can assume that $g \in A(L)$ and $h \in A(K)$. This yields a special case of a result of $[5]$.

3.3. Universal Dirichlet series and unbounded operators. In this section, we consider ordinary Dirichlet series $\sum_{n=1}^{+\infty} a_{n} n^{-z}$ absolutely convergent for $\Re(z)>0$.

Definition 3.6. A compact set $K \subset \mathbb{C}$ is called admissible for Dirichlet series if $K^{c}$ is connected and $K=K_{1} \cup \cdots \cup K_{d}$, where each $K_{i}$ is contained in a strip $\left\{z \in \mathbb{C}: c_{i} \leq \Re(z) \leq b_{i}\right\}$ with $b_{i}-c_{i}<1 / 2$, the strips being disjoint.

Let $1<p<+\infty$ and set $A=\ell^{p}$, the Banach space endowed with the norm $\|a\|_{p}=\left(\sum_{j \geq 1}\left|a_{j}\right|^{p}\right)^{1 / p}$. We fix a compact set $K \subset\{z \in \mathbb{C}: \Re(z) \leq 0\}$ admissible for Dirichlet series. Consider the space $X=H(\mathbb{C})$ of entire functions equipped with the metric $\rho(f, g)=\sup _{K}|f-g|, f, g \in H(\mathbb{C})$. Define

$$
T: A \rightarrow A, \quad a=\left(a_{1}, a_{2}, a_{3}, \ldots\right) \mapsto\left(-\log (2) a_{2},-\log (3) a_{3}, \ldots\right) .
$$

For any $n \in \mathbb{N}^{*}$, we denote $T_{n}=T^{n}$. Observe that $T$ can be viewed as the operator $S\left(\partial / \partial z\left(\sum_{n \geq 1} a_{n} n^{-z}\right)\right)$ where $S$ is the backward shift. Clearly the set of Dirichlet polynomials is a dense subset of the domain of $T_{n}$ and for every $a \in G, T_{n} a$ converges to zero. Moreover, for any $n \in \mathbb{N}^{*}, T_{n}$ is an unbounded and closed linear operator. We need a result on approximation by Dirichlet polynomials. 
Lemma 3.7. Let $K \subset\{z \in \mathbb{C}: \Re(z) \leq 0\}$ be an admissible compact set. Let $g: K \rightarrow \mathbb{C}$ be a function continuous on $K$ and holomorphic in $\stackrel{\circ}{K}$, and let $\varepsilon>0, l \in \mathbb{N}$ and $q \in \mathbb{N}$. Then there exists a Dirichlet polynomial $h(z)=\sum_{j=q+1}^{m} a_{j} j^{-z}$ such that $\sup _{z \in K}|h(z)-g(z)|<\varepsilon$ and $\left(\sum_{j=q+1}^{m}\left|\log ^{l}(j) a_{j}\right|^{p}\right)^{1 / p}<\varepsilon$.

Proof. This follows from Lemma 2 of [1] by taking $f=0$ with slight modifications. Indeed, we may suppose that $g$ is an entire function, by Mergelyan's Theorem. Write $K=K_{1} \cup \cdots \cup K_{d}$ with $K_{i} \subset\{z \in \mathbb{C}$ : $\left.c_{i} \leq \Re(z) \leq b_{i}\right\}$, and $0 \geq b_{1} \geq c_{1}>b_{2} \geq \cdots>b_{d} \geq c_{d}, b_{i}-c_{i}<1 / 2$. We fix $\sigma_{1} \in \mathbb{R}$ such that $K_{1}+\sigma_{1} \subset\{z \in \mathbb{C}: 1 / 2<\Re(z)<1\}$ and $\sigma_{1} p>1$. Consider $n_{1} \geq q$ such that $\sum_{j \geq n_{1}+1}\left(\log ^{l}(j) j^{-\sigma_{1}}\right)^{p}<(\varepsilon / 2)^{p}$. We end as in the proof of Lemma 2 of [1, p. 174].

Theorem 3.8. Let $A$ be an $\ell^{p}, 1<p<+\infty$. Under the above assumptions, there exists a sequence $a=\left(a_{n}\right) \in A$ such that for every $b \in A$ and for every entire function $h$ there exists $\left(\lambda_{n}\right) \in \mathbb{N}^{\mathbb{N}}$ such that $T_{\lambda_{n}}(a) \rightarrow b$ and $\sup _{z \in K}\left|\sum_{j=1}^{\lambda_{n}} a_{j} j^{-z}-h(z)\right| \rightarrow 0$ as $n \rightarrow+\infty$. The set of such sequences is dense in $A$.

Proof. It suffices to prove that condition (ii') of Theorem 2.12 holds. Let $q \in \mathbb{N}$ and $\varepsilon>0$. By Lemma 3.7, there exist integers $n, p$, with $n \geq p \geq q$, and complex numbers $a_{p}, \ldots, a_{n}$ such that $\sup _{z \in K} \mid \sum_{j=p}^{n} a_{j} j^{-z}-$ $g(z) \mid<\varepsilon$ and $\left(\sum_{j=p}^{n}\left|\log ^{q}(j) a_{j}\right|^{p}\right)^{1 / p}<\varepsilon / 2$. We fix $N \in \mathbb{N}$ such that $\left(\sum_{j \geq N+1}\left|b_{j}\right|^{p}\right)^{1 / p}<\varepsilon$. Let $b^{\prime}=\left(b_{1}, \ldots, b_{N}, 0, \ldots\right)$. Then $\left\|b-b^{\prime}\right\|_{p}<\varepsilon$. Moreover, choose an integer $k>n$ so that

$$
\left(\sum_{j=1}^{N} \frac{\left|b_{j}\right|^{p}}{\log ^{p}(j+1) \ldots \log ^{p}(j+k-i)}\right)^{1 / p}<\varepsilon / 2, \quad i=0,1, \ldots, q .
$$

We set $m=N+k$ and $c=\left(c_{j}\right)_{j=1}^{\infty}$, with

$$
c_{j}= \begin{cases}0 \quad \text { for } j \in\{1, \ldots, p-1\} \cup\{n+1, \ldots, k\} \cup\{m+1, m+2, \ldots\}, \\ a_{j} \quad \text { for }=p, \ldots, n, \\ \frac{(-1)^{k} b_{j-k}}{\log (j+1-k) \ldots \log (j)} \quad \text { for } j=k+1, \ldots, m .\end{cases}
$$

Then we get

$$
\begin{gathered}
\sup _{z \in K}\left|\sum_{j=p}^{k} c_{j} j^{-z}-g(z)\right|=\sup _{z \in K}\left|\sum_{j=p}^{n} a_{j} j^{-z}-g(z)\right|<\varepsilon \\
\left(\sum_{j=p}^{k}\left|c_{j}\right|^{p}\right)^{1 / p}=\left(\sum_{j=p}^{n}\left|a_{j}\right|^{p}\right)^{1 / p}<\varepsilon
\end{gathered}
$$




$$
\begin{gathered}
\left(\sum_{j=k+1}^{m}\left|c_{j}\right|^{p}\right)^{1 / p}=\left(\sum_{j=1}^{N} \frac{\left|b_{j}\right|^{p}}{\log ^{p}(j+1) \ldots \log ^{p}(j+k)}\right)^{1 / p}<\varepsilon, \\
\left\|T_{k}(c)-b\right\|_{p}=\left\|b^{\prime}-b\right\|_{p}<\varepsilon .
\end{gathered}
$$

Finally, the following estimate holds, for every $i=1, \ldots, q$ :

$$
\begin{aligned}
\left\|T_{i}(c)\right\|_{p} & \leq\left(\sum_{j=p}^{n}\left|\log ^{q}(j) a_{j}\right|^{p}\right)^{1 / p}+\left(\sum_{j=1}^{N} \frac{\left|b_{j}\right|^{p}}{\log ^{p}(j+1) \ldots \log ^{p}(j+k-i)}\right)^{1 / p} \\
& <\varepsilon / 2+\varepsilon / 2=\varepsilon .
\end{aligned}
$$

Therefore condition (ii') of Theorem 2.12 is satisfied.

Let us recall that there exists a sequence $\left(K_{k}\right)$ of admissible compact sets contained in $\{z \in \mathbb{C}: \Re(z) \leq 0\}$ such that every admissible compact set $K \subset\{z \in \mathbb{C}: \Re(z) \leq 0\}$ is contained in some $K_{k}$ (see [2, Lemma 5]). We combine the previous result with Theorem 2.14 and Mergelyan's Theorem to obtain the following.

Theorem 3.9. Let $A$ be an $\ell^{p}, 1<p<+\infty$. Under the above assumptions, there exists a sequence $a=\left(a_{n}\right) \in A$ such that, every $b \in A$, for every admissible compact set $K \subset\{z \in \mathbb{C}: \Re(z) \leq 0\}$ and every function $h$ continuous on $K$ and holomorphic in $\stackrel{\circ}{K}$ there exists $\left(\lambda_{n}\right) \in \mathbb{N}^{\mathbb{N}}$ such that $T_{\lambda_{n}}(a) \rightarrow b$ and $\sup _{z \in K}\left|\sum_{j=1}^{\lambda_{n}} a_{j} j^{-z}-h(z)\right| \rightarrow 0$ as $n \rightarrow+\infty$. The set of such sequences is dense in $A$.

Acknowledgements. Many thanks to V. Nestoridis for helpful comments.

\section{References}

[1] F. Bayart, Topological and algebraic genericity of divergence and universality, Studia Math. 167 (2005), 161-181.

[2] F. Bayart, K.-G. Grosse-Erdmann, V. Nestoridis and C. Papadimitropoulos, Abstract theory of universal series and applications, Proc. London Math. Soc. 96 (2008), 417-463.

[3] J. Bès, K. C. Chan and S. M. Seubert, Chaotic unbounded differentiation operators, Integral Equations Operator Theory 40 (2001), 257-267.

[4] G. Costakis, V. Nestoridis and V. Vlachou, Smooth univalent universal functions, Math. Proc. Roy. Irish Acad. 107 (2007), 101-114.

[5] G. Costakis and V. Vlachou, Identical approximative sequence for various notions of universality, J. Approx. Theory 132 (2005), 15-24.

[6] O. Demanze and A. Mouze, On universal formal power series, J. Math. Anal. Appl. 338 (2008), 662-674.

[7] K.-G. Grosse-Erdmann, Universal families and hypercyclic operators, Bull. Amer. Math. Soc. 36 (1999), 345-381. 
A. Mouze

[8] W. Luh, Approximation analytischer Funktionen durch überkonvergente Potenzreihen und deren Matrix-Transformierten, Mitt. Math. Sem. Giessen 88 (1970), 1-56.

[9] V. Nestoridis, Universal Taylor series, Ann. Inst. Fourier (Grenoble) 46 (1996), $1293-1306$.

[10] V. Nestoridis and C. Papadimitropoulos, Abstract theory of universal series and an application to Dirichlet series, C. R. Math. Acad. Sci. Paris 341 (2005), 539-543.

[11] A. I. Seleznev, On universal power series, Mat. Sb. 28 (1951), 453-460 (in Russian).

A. Mouze

Laboratoire Paul Painlevé, UMR 8524

Current address:

École Centrale de Lille

Cité Scientifique

59650 Villeneuve d'Ascq, France

E-mail: Augustin.Mouze@math.univ-lille1.fr

Received February 4, 2009

Revised version May 28, 2009 